\title{
THEORETICAL STUDY OF ABSORPTION OF LIGHT DUE TO EFFECT OF PONDEROMOTIVE FORCE
}

\section{P. K. Thakur and J. J. Nakarmi}

Journal of Nepal Physical Society

Volume 5, Issue 1, October 2019

ISSN: $2392-473 X$

Editors:

Dr. Vinaya Kumar Jha

Dr. Binod Adhikari

Dr. Kapil Adhikari

JNPS, 5 (1), 91-96 (2019)

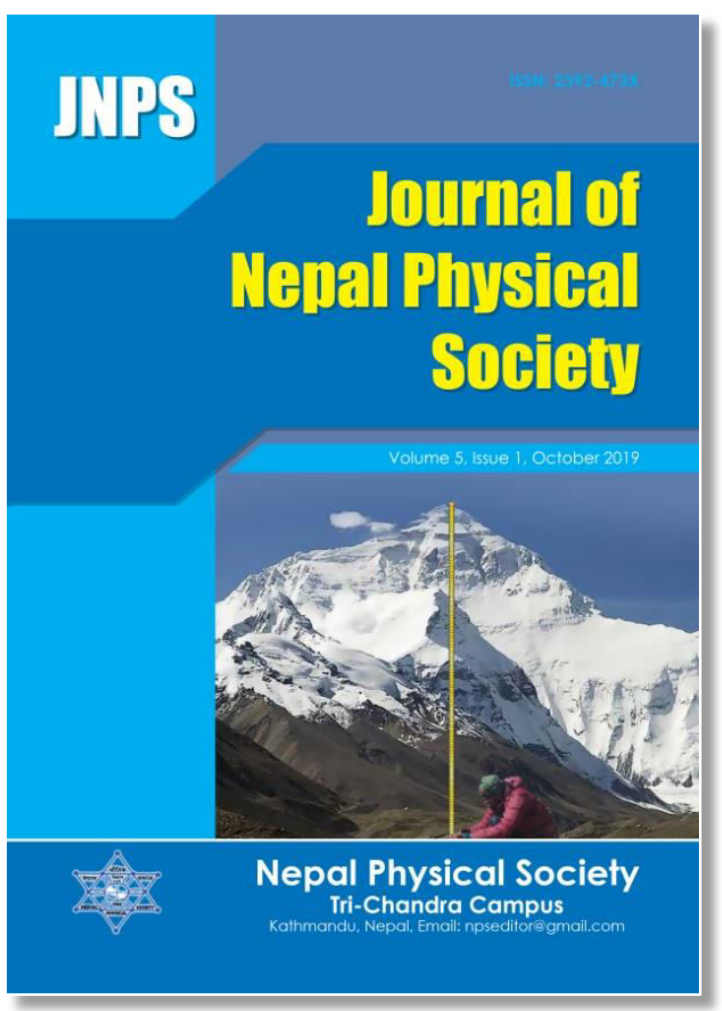

Published by:

Nepal Physical Society

P.O. Box: 2934

Tri-Chandra Campus

Kathmandu, Nepal

Email:npseditor@gmail.com 


\title{
THEORETICAL STUDY OF ABSORPTION OF LIGHT DUE TO EFFECT OF PONDEROMOTIVE FORCE
}

\author{
P. K. Thakur* and J. J. Nakarmi \\ Central Department of Physics, T.U., Kirtipur, Nepal \\ *Corresponding Email: pk_thakur12@yahoo.com
}

\begin{abstract}
Absorption of light due to effect of ponderomotive force in laser plasma interaction which has seen that the nonlinear interaction results from rigorous application of the ponderomotive force description based on Lorentz's theory. The deduced equation of motion is more general than that of the two-fluid model of the plasma and that used in the theory of microwave interaction with plasma. As might be expected, the forces are only in the direction of lower plasma densities and tangential forces vanish only with the general equation of two-fluid model. This result has been verified upto the third order in the spatial variation of the electron density. In addition it is seen that the collision frequency decreases continuously with the increase in temperature. From these results, it is concluded that the absorption coefficient decreases continuously with the increase in temperature. Furthermore, this work describes the variation of the absorption coefficient with laser light frequency and shows that the absorption coefficient depends on the frequency of light.
\end{abstract}

Keywords: Absorption, Ponderomotive Force, Laser Light Frequency.

\section{INTRODUCTION}

\section{Background of the Study}

Theoretical study on absorption of light due to effect of Ponderomotive force in the interaction of laser radiation with plasma has been discussed from several points of view. Plasma heating as the result of laser light absorption means of the usual collision processes $[1,3,5]$. The macroscopic motion of plasma due to the interaction of the electromagnetic field $[2,6]$. In the numerical simulations have revealed extremely high self generated magnetic fields in the interaction of ultraintense laser pulse with over dense plasma target [3, 18]. The generation of such extremely high magnetic fields is the temporal variations of the ponderomotive force of the laser light on the plasma [10].

In order to study the propagation of laser light in plasma and self generation of magnetic field by resonance absorption of laser light we have to solve the wave equation. The wave equation is obtained from Maxwell's equations by usual procedure. In this case we consider Maxwell's equations, in addition to poynting vector $[8,19]$.

\section{Maxwell's Equations and Wave Equation} In Maxwell's equations in Gaussian Unit are,

$$
\begin{aligned}
& \nabla \cdot \vec{D}=4 \pi \rho \\
& \nabla \times \overrightarrow{\mathrm{E}}=-\frac{1}{c} \frac{\partial \overrightarrow{\mathrm{B}}}{\partial t} \\
& \nabla \cdot \overrightarrow{\mathrm{B}}=0 \\
& \nabla \times \overrightarrow{\mathrm{B}}=\frac{4 \pi \vec{J}}{c}+\frac{1}{c} \frac{\partial \vec{D}}{\partial t}
\end{aligned}
$$

From the above equations we get homogeneous form of equation in the medium $(\nabla \in=0)[4,14]$

$$
\nabla^{2} \overrightarrow{\mathrm{B}}-\frac{1}{\epsilon_{r}} \nabla \in_{r} \cdot \nabla \overrightarrow{\mathrm{B}}+\frac{\omega^{2} \epsilon_{r}}{c^{2}} \overrightarrow{\mathrm{B}}=0
$$

\section{Poynting vector}

For the single charge q moving with velocity $\vec{v}$ in the external electromagnetic fields $\overrightarrow{\mathbf{E}}$ and $\overrightarrow{\mathrm{B}}$ the rate of work done by the fields is $\mathrm{q} \vec{v} . \vec{E}$. Here it should be noted that work done by the magnetic field is zero, since magnetic force is always perpendicular to the velocity. If there exist a continuous distribution of charge and current, the total rate of doing work by the field in the finite volume $\mathrm{V}$ is, 


$$
W=\int_{V} \vec{J} \cdot \overrightarrow{\mathrm{E}} d^{3} x
$$

Continuity equations or conservation law since the volume $\mathrm{V}$ is arbitrary; this can be cast into the form of differential [9]

$$
\frac{\partial \vec{u}}{\partial t}+\nabla \cdot \vec{S}=-\vec{J} \cdot \overrightarrow{\mathrm{E}}
$$

Where the vector $\mathrm{S}$ is given by

$$
S=(\overrightarrow{\mathrm{E}} \times \overrightarrow{\mathrm{H}})
$$

and is called Poynting vector.

This vector $S$ is interpreted as the amount of field energy passing normally through unit area of boundary surface enclosing the source in unit time. Hence, dimension of $S$, is (energy per unit area per unit time). Since only its divergence appears in the conservation law, the poynting vector seems arbitrary to the extent that the curl of any vector field can be added to it. Such added term can however have no physical consequences.

After solving we get the expression of Maxwell Stress Tensor $[10,19]$

$$
\mathrm{T}_{\alpha \beta}=\epsilon_{o}\left[\overrightarrow{\mathrm{E}}_{\alpha} \overrightarrow{\mathrm{E}}_{\beta}+\overrightarrow{\mathrm{B}}_{\alpha} \overrightarrow{\mathrm{B}}_{\beta}-\frac{1}{2}(\overrightarrow{\mathrm{E}} \cdot \overrightarrow{\mathrm{E}}+\overrightarrow{\mathrm{B}} \cdot \overrightarrow{\mathrm{B}}) \delta_{\alpha \beta}\right](5)
$$

Where $\mathrm{T}_{\alpha \beta}$ is the Maxwell's stress tensor.

\section{Ponderomotive Force}

The ponderomotive force acts primarily on the electron due to their small mass and is transmitted to the ions by the condition of charge neutrality. Hence, the momentum carried by the electromagnetic wave can be transferred directly to the plasma by means of this force [3]. The general form of this force [15]

$$
\vec{F}_{p}=-\frac{e^{2}}{4 m_{e} \omega^{2}} \nabla \vec{E} \vec{E}^{*}
$$

which is derived by using the equation with,

$$
\vec{f}=\nabla \cdot \sigma-\frac{\partial}{\partial t}\left(\frac{\overrightarrow{\mathrm{E}} \times \overrightarrow{\mathrm{H}}}{4 \pi c}\right)
$$

Where, $\vec{f}$ is the force density in plasma in the presence of electric and magnetic field $\overrightarrow{\mathrm{E}}$ and $\overrightarrow{\mathrm{H}}$. $\sigma$ is the Stress Tensor of Plasma, $\mathrm{t}$ is time and $\mathrm{c}$ is the velocity of light. This relation is also defined for the equation of motion [10].

\section{ABSORPTION COEFFICIENT}

\section{Basic Equations}

The momentum acquired by electrons in plasma in the pressure of electro-magnetic wave is

$$
m_{e} \frac{\partial \vec{v}}{\partial t}=-e \vec{E}+\vec{F}_{p}+\frac{1}{n_{e}} \nabla n_{e} T_{e}-m_{e} v_{e i} \vec{v}
$$

where $\mathrm{m}_{\mathrm{e}}, \mathrm{n}_{\mathrm{e}}, \mathrm{T}_{\mathrm{e}}$ are mass number, density temperature and charge of electron, $\mathrm{B}$ is the magnetic field of the wave, $\vec{E}$ is the total electric field which is equal to sum of the electric field of the wave $\overrightarrow{\mathrm{E}}$ and the space charge field $\overrightarrow{\mathrm{E}}$ and $\overrightarrow{F_{p}}$ is Ponderomotive force, $v_{\mathrm{ei}}$ is the electron- ion collision frequency $[1,3]$.

Considering the space parts, which has slow variation [16], we have,

$T_{e} \frac{\nabla n_{e}}{n_{e}}=-e \vec{E}_{s}+\vec{F}_{p}-\nabla T_{e}$

\section{Determination of current density}

Now, the induced current $\vec{J}$, when the electromagnetic wave propagates through the plasma, can be written as,

$$
\mu=-n_{e} e v
$$

From above equations, we obtain,

$$
\begin{aligned}
\vec{J} & =\frac{\nabla n_{e}}{F} \frac{1}{l_{n}}\left(1-\frac{Z}{L}\right) e \frac{e \vec{E}}{m_{e}\left(i \omega+v_{e i}\right)} \\
& =\frac{\nabla n_{e}}{F} \frac{1}{l_{n}}\left(1-\frac{Z}{L}\right) \frac{e^{2}}{m_{e}\left(i \omega+v_{e i}\right)} \vec{E} \\
& =\frac{\nabla n_{e}}{F} \frac{1}{l_{n}}\left(1-\frac{Z}{L}\right) \frac{e^{2}}{m_{e}}\left[\frac{\left(-i \omega+v_{e i}\right)}{\left(\omega^{2}+v_{e i}^{2}\right)}\right] \vec{E} \\
& =\frac{\nabla n_{e}}{F} \frac{1}{l_{n}}\left(1-\frac{Z}{L}\right) \frac{e^{2}}{m_{e}}\left[\frac{\left(v_{e i}-i \omega\right)}{\left(\omega^{2}+v_{e i}{ }^{2}\right)}\right] \vec{E}
\end{aligned}
$$

The above equation represents current induced by Ponderomotive force, which gives rise to magnetic field. 


\section{For the calculation of absorption coefficient}

We can write the equation in the form

$$
\begin{aligned}
\vec{J} \cdot \vec{E} & =\frac{\nabla n_{e}}{F} \frac{1}{l_{n}}\left(1-\frac{Z}{L}\right) \frac{e^{2}}{m_{e}}\left[\frac{\left(v_{e i}-i \omega\right)}{\left(\omega^{2}+v_{e i}{ }^{2}\right)}\right] \vec{E} \cdot \overrightarrow{E^{*}} \\
& =\frac{\nabla n_{e}}{F} \frac{1}{l_{n}}\left(1-\frac{Z}{L}\right) \frac{e^{2}}{m_{e}} \frac{1}{\left(\omega^{2}+v_{e i}{ }^{2}\right)}\left\{v_{e i}-i \omega\right\} \vec{E} \cdot \vec{E}^{*} \\
& =\frac{\nabla n_{e}}{F} \frac{1}{l_{n}}\left(1-\frac{Z}{L}\right) \frac{e^{2}}{m_{e}} \frac{v_{e i}}{\left(\omega^{2}+v_{e i}{ }^{2}\right)} \vec{E} \cdot \overrightarrow{E^{*}}-i \frac{\nabla n_{e}}{F} \frac{1}{l_{n}}\left(1-\frac{Z}{L}\right) \frac{e^{2}}{m_{e}} \frac{\omega}{\left(\omega^{2}+v_{e i}{ }^{2}\right)} \vec{E} \cdot \vec{E}^{*}
\end{aligned}
$$

We know that the average value of energy absorbed per unit time per unit volume $P_{a v}$ through joule heating $[3,13]$ is given by,

$$
P_{a v}=-\frac{1}{2} \operatorname{Re}[\vec{J} \cdot \vec{E}]
$$

From above Eqn $(11,12)$, we get

$$
P_{a v}=-\frac{1}{2} \frac{\nabla n_{e}}{F} \frac{1}{l_{n}}\left(1-\frac{Z}{L}\right) \frac{e^{2}}{m_{e}} \frac{v_{e i}}{\left(\omega^{2}+v_{e i}{ }^{2}\right)} \vec{E} \cdot \overrightarrow{E^{*}}
$$

We know that the poynting vector is defined as

$$
S=\frac{1}{8 \pi} c \operatorname{Re}\left(\frac{c k}{\omega}\right) \vec{E} \cdot \vec{E}^{*}
$$

So that

$$
\vec{E} \cdot \overrightarrow{E^{*}}=\frac{8 \pi S}{c \operatorname{Re}\left(\frac{c k}{\omega}\right)}
$$

From Eqns (13) and (14) we get,

$$
P_{a v}=-\frac{1}{2} \frac{\nabla n_{e}}{F} \frac{1}{l_{n}}\left(1-\frac{Z}{L}\right) \frac{e^{2}}{m_{e}} \frac{v_{e i}}{\left(\omega^{2}+v_{e i}{ }^{2}\right)} \frac{8 \pi S}{c \operatorname{Re}\left(\frac{c k}{\omega}\right)}
$$

If the size of the plasma is small then we get,

$$
P_{a v}=\frac{4 \pi m_{e} e^{2}}{m_{e}} \frac{\nabla n_{e}}{n_{e} F} \frac{Z}{l_{n}} \frac{v_{e i}}{\left(\omega^{2}+v_{e i}^{2}\right)} \frac{S}{c \operatorname{Re}\left(\frac{c k}{\omega}\right)}
$$

From Eqn(10) with the dispersion relation, the wave equation for electric vector, so that Eqn (16) becomes

$$
\begin{aligned}
& P_{a v}=\frac{4 \pi n_{e} e^{2}}{m_{e}} \frac{\nabla n_{e}}{n_{e} F} \frac{Z}{l_{n}} \frac{v_{e i}}{\left(\omega^{2}+v_{e i}{ }^{2}\right)} \frac{1}{c} \frac{1}{\left\{1+\left|\frac{\nabla n_{e}}{n_{e}}\right| \frac{1}{l_{n} F}\left(1-\frac{Z}{L}\right)\left[\frac{\omega_{p}^{2}}{\left(\omega^{2}+v_{e i}{ }^{2}\right)}\right]\right\}^{\frac{1}{2}} S} \\
& =\frac{1}{c} \frac{\nabla n_{e}}{n_{e} F} \frac{Z}{l_{n}} \frac{\omega_{p}{ }^{2} v_{e i}}{\left(\omega^{2}+v_{e i}{ }^{2}\right)}\left\{1+\left|\frac{\nabla n_{e}}{n_{e}}\right| \frac{1}{l_{n} F}\left(1-\frac{Z}{L}\right)\left[\frac{\omega_{p}^{2}}{\left(\omega^{2}+v_{e i}{ }^{2}\right)}\right]\right\}^{-\frac{1}{2}} S
\end{aligned}
$$

The absorption coefficient $(\alpha)$ is then given by the coefficient of $\mathrm{S}$ (poynting vector) of Eqn (17), then we get

$\alpha=\frac{1}{c} \frac{\nabla n_{e}}{n_{e} F} \frac{Z}{l_{n}} \frac{\omega_{p}^{2} v_{e i}}{\left(\omega^{2}+v_{e i}{ }^{2}\right)}\left\{1+\left|\frac{\nabla n_{e}}{n_{e}}\right| \frac{1}{l_{n} F}\left(1-\frac{Z}{L}\right)\left[\frac{\omega_{p}^{2}}{\left(\omega^{2}+v_{e i}{ }^{2}\right)}\right]\right\}^{-\frac{1}{2}}$

This is the required expression for the absorption coefficient. 


\section{RESULT AND DISCUSSION}

The Ponderomotive force has verified the existence of substantial macroscopic acceleration of inhomogeneous plasma by interaction with laser light, and its description based on Lorentz's theory

$$
\mathrm{f}=-\nabla \cdot \overrightarrow{\mathrm{P}}+\nabla \cdot\left[\mathrm{U}-\frac{\omega_{\mathrm{p}}^{2}}{4 \pi\left(\omega^{2}+v^{2}\right)}\left(1+\mathrm{i} \frac{v}{\omega}\right) \overrightarrow{\mathrm{E}} \overrightarrow{\mathrm{E}}\right]-\frac{\partial}{\partial \mathrm{t}} \frac{\mathrm{E} \times \overrightarrow{\mathrm{H}}}{4 \pi \mathrm{c}}
$$

It can be deduced according to the macroscopic theory of the two -fluid model with [12], we get

$$
\mathrm{f}=-\nabla \cdot \overrightarrow{\mathrm{P}}+\frac{1}{\mathrm{c}} \overrightarrow{\mathrm{J}} \times \overrightarrow{\mathrm{H}}+\overrightarrow{\mathrm{J}} \cdot \nabla \frac{1}{\omega^{2}} \frac{\partial \overrightarrow{\mathrm{E}}}{\partial \mathrm{t}}
$$

When the non-linear collisionless acceleration of inhomogeneous plasma at oblique incidence of light is calculated then the necessity of using all nonlinear terms of the general equation of motion is demonstrated [7]. The forces are only in the direction of lower plasma densities and tangential forces vanish only with the general condition When high-powered microwaves or laser beams are used to heat or confine plasmas, however, the radiation pressure can reach several hundred thousand atmospheres, when applied to plasma, this force is compiled to the particles in a somewhat subtle way and is called the Ponderomotive force. Many nonlinear phenomena have a simple explanation in terms of the Ponderomotive force [7].

The simplest way to derive this nonlinear force is to consider the motion of an electron in the oscillating electric and magnetic fields of wave. Thus, the formula for Ponderomotive force in the general form is

$$
\vec{F}_{p}=-\frac{e^{2}}{4 m_{e} \omega^{2}} \nabla \vec{E} \vec{E}^{*}
$$

Thus the dynamics of the laser - plasma interaction may be described mathematically by the onedimensional two-fluid conservation equations of
[11]. The deduced equation of motion is more general than that of the two -fluid model of the plasma and that used in the theory of microwave interaction with plasma which can be represented by the following form , mass, density, momentum (with the Ponderomotive force) and energy, the sharp laser intensity gradient created a Ponderomotive force oppositely directed to the expansion. After using the basic equation of the momentum acquired by electrons in plasma in the presence of electromagnetic wave the value of the number of electron density $\left(n_{e}\right)[16,17]$.

$n_{e}=-\frac{\nabla n_{e}}{F} \frac{1}{l_{n}}\left(1-\frac{Z}{L}\right)$

where,

$$
F=-\left\{\frac{T_{i}}{T_{e}} \frac{1}{l_{n_{i}}}+\frac{e^{2}}{4 m_{e} \omega^{2} T_{e}} \nabla \vec{E} \stackrel{\vec{E}}{E^{*}}+\frac{1}{l T_{e}}\right\}
$$

Thus after calculating the current induced by Ponderomotive force, when the electromagnetic wave propagates through the plasma is

$$
\vec{J}=\frac{\nabla n_{e}}{F} \frac{1}{l_{n}}\left(1-\frac{Z}{L}\right) \frac{e^{2}}{m_{e}}\left[\frac{\left(V_{e i}-i \omega\right)}{\left(\omega^{2}+V_{e i}^{2}\right)}\right] \vec{E}
$$

By incorporating this equation with Maxwell's Equation, the value of effective dielectric constant is found [3, 17]. Further using the induced current the conductivity is calculated which is represented by the equation

$$
n^{2}=\left(\frac{k c}{\omega}\right)^{2}=1+i \frac{4 \pi \sigma}{\omega}
$$

So that, the value of refractive index is

$$
\eta=\operatorname{Re}\left(\frac{c k}{\omega}\right)=\left\{1+\left|\frac{\nabla n_{e}}{n_{e}}\right| \frac{1}{l_{n} F}\left(1-\frac{Z}{L}\right)\left[\frac{\omega_{p}^{2}}{\left(\omega^{2}+V_{e i}^{2}\right)}\right]\right\}^{\frac{1}{2}}
$$

After calculating the refractive index by using the theorem of poynting vector and also using average value of energy absorbed per unit volume through joule heating, we have calculated the absorption coefficient i.e.

$$
\alpha=\frac{1}{c} \frac{\nabla n_{e}}{n_{e} F} \frac{Z}{l_{n}} \frac{\omega_{p}^{2} V_{e i}}{\left(\omega^{2}+V_{e i}^{2}\right)}\left\{1+\left|\frac{\nabla n_{e}}{n_{e}}\right| \frac{1}{l_{n} F}\left(1-\frac{Z}{L}\right)\left[\frac{\omega_{p}^{2}}{\left(\omega^{2}+V_{e i}^{2}\right)}\right]\right\}^{-\frac{1}{2}}
$$


In fig (1) the graph between $\mathrm{F}$ and frequency with the help of Eqn (22) are cleared that if the value of frequency increases then the value of $\mathrm{F}$ going to be decreases. It is also cleared that at the minimum value of frequency the value of $F$ becomes maximum. In the same way in fig (2) the graph between the absorption coefficient and frequency with the help of Eqn (18) it is cleared that if the value of absorption coefficient decreases but it does not tend to zero. It is also cleared that if the value of frequency is minimum then absorption coefficient is maximum. In fig(3) the graph between absorption coefficient and temperature in $\mathrm{eV}$ with the help of Eqns (18) and (23) it is cleared that if the temperature of the laser light increases then the value of absorption coefficient is going to be decreases. If the value of temperature is minimum then the value of absorption coefficient is maximum.

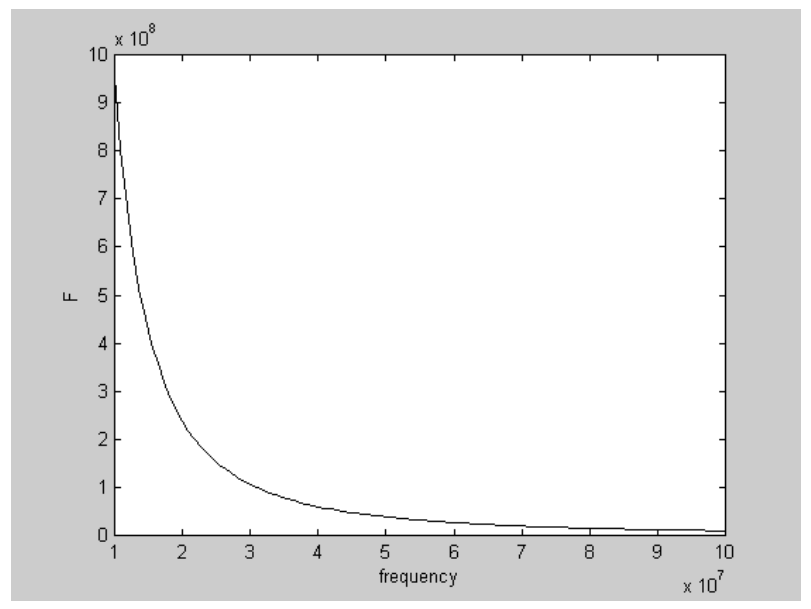

Fig. 1: The variation of $F$ with frequency.

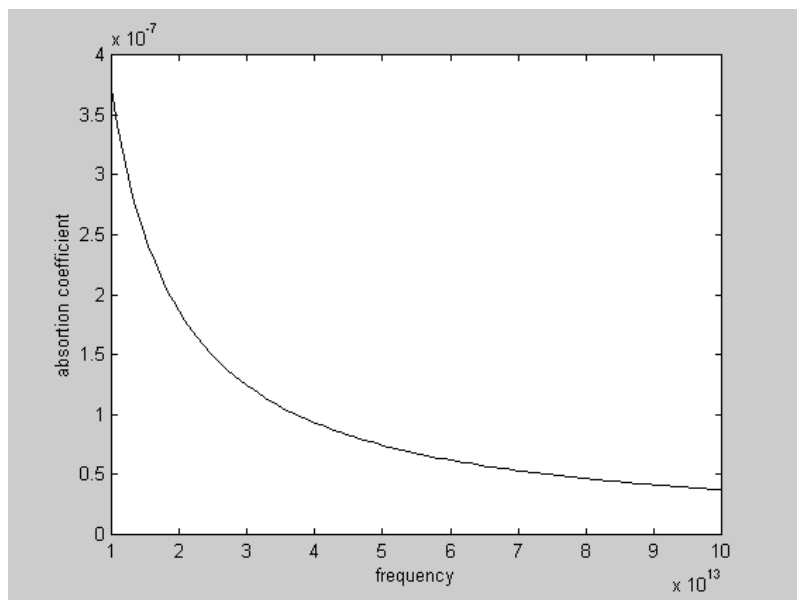

Fig. 2: The Variation of absorption coefficient with frequency.

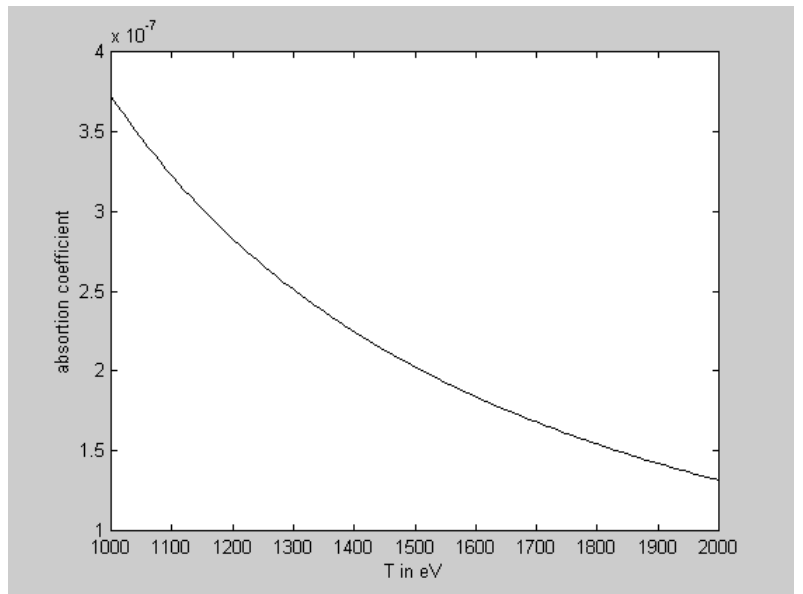

Fig. 3: The Variation of absorption coefficient with Temperature in $\mathrm{eV}$.

\section{CONCLUSION}

For the study of absorption of light due to effect of Ponderomotive force in laser plasma interaction are concluded that the Ponderomotive force has verified the existence of substantial macroscopic acceleration of an inhomogeneous plasma by interaction with laser light and is explained according as Lorentz's theory. At oblique incidence, all the nonlinear terms of the general equation of motion is demonstrated. It is also concluded that the forces are only in the direction of lower plasma densities. The nonlinear acceleration is in the direction of the laser light due to the electron density is equal to or higher than that which gives a plasma frequency in which the temperature - dependent upper limit of the electron densities below. The nonlinear absorption coefficient based on the recoil momentum and is also dependence upon the temperature above $130 \mathrm{eV}$. In fig (1) the graph between $\mathrm{F}$ and frequency $(\omega)$ is cleared that if the value of frequency increases then the value of $F$ going to be decreases. In fig (2) the graph between absorption coefficient $(\alpha)$ and frequency $(\omega)$ is cleared that if the value of frequency increases then the value of absorption coefficient going to be decreases. In fig (3) the graph between absorption coefficient $(\alpha)$ and temperature $(\mathrm{T})$ in $\mathrm{eV}$ shows that if the value of temperature increases then the value of absorption coefficient going to be decreases.

\section{REFERENCES}

[1] F.F. Chen, Introduction to Plasma Physics and Controlled Fusion, Vol-I, Second edition, Plenum Press, New York (1984). 
[2] V.L. Ginberg, The Propagation of Electromagnetic Waves in Plasma, second edition, Pergamon Oxford, (1970).

[3] J.J. Nakarmi, L.N. Jha, Effect of Ponderomotive Force on Self-generation of Magnetic Field and Absorption Coefficient in Laser Plasma,Proceedings of III National Conference on IoST, March, 8-11, 1999.

[4] N. G. Basov and O.N. Krokhin, Phys. Fluids, 46, 171 (1964).

[5] J. M. Dawson and C. Oberman, Phys. Fluids, 5, 517, (1962).

[6] W. I. Linlor, Phys Rev, 12, 383 (1964).

[7] H. Hora, D. Pfirsch, and A. Schluter, Z. Naturforsch, Phys. Fluids, 22a, 278 (1967).

[8] H. Mofz and C. J. H. Watson, Advances in Electronics and Electron Physics, 23, 153, (1967).

[9] R. Spiegel, Theory and Problems of Vector Analysis, McGraw-Hill Publishing Co. Ltd. New Delhi,p.166,(1974).
[10] L.D. Landau, and E.M. Lifshitz, Electrodynamics of continuous Media, p. 242, (1966).

[11] H. Hora, Opto. Electronics 2, 202 (1970).

[12] S. Rand, Phys. Rev. B136, 231 (1964).

[13] Aliev, Y.M. and V.Y. Bychenkov, Sov. J. Plasma Phys, 6(1), 46 (1980).

[14] Borgesi, M.A. J. Mackinnon, R. Gaillard, O.Willi, A. Pukhov and J. Maeyer-ter-Vehn, Phys. Rev. Lett., 88, 5137 (1998).

[15] Sodha, M. S., A. K. Ghatak and V. K. Tripathi. Self - Focusing of Laser Beams, Tata McGrawHill Publishing Co. Ltd. New Delhi, p. 82, (1974).

[16] Sudan, R. N., Phys. Rev. Lett., 70, 3075 (1993).

[17] Washim, H. and V. K. Karpman, Sov. Phys. JETP 44(a), 528 (1976).

[18] Wilks, S. C., W.L.Kruer,M.Tabak and A.B. Langdon et al. Phys. Rev. Lett. 69,1383, (1992).

[19] S.P. Puri, Classical Electrodynamics, Alpha Science International Ltd., United Kingdom (1993). 